
Not for reproduction, distribution or commercial use.

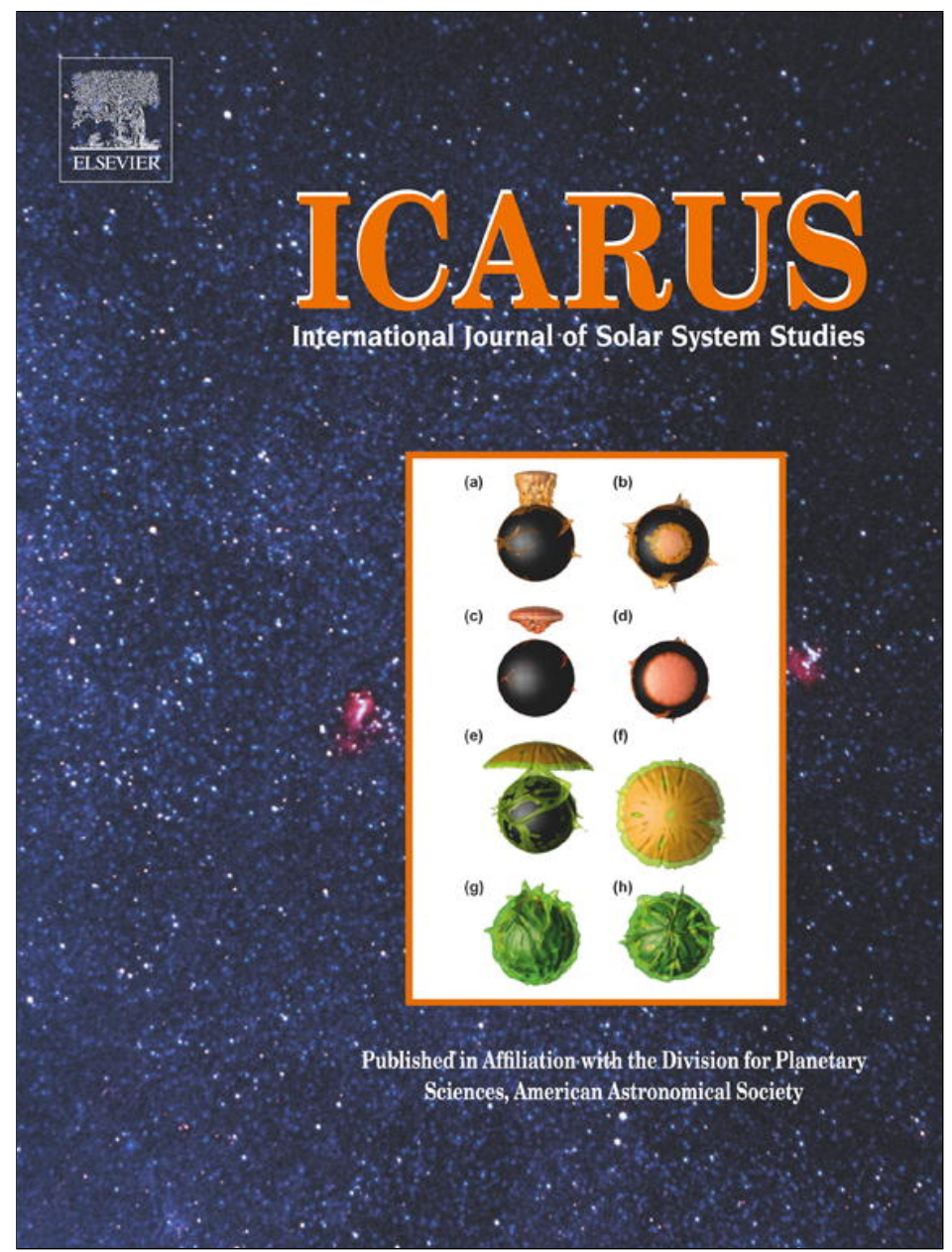

This article appeared in a journal published by Elsevier. The attached copy is furnished to the author for internal non-commercial research and education use, including for instruction at the authors institution and sharing with colleagues.

Other uses, including reproduction and distribution, or selling or licensing copies, or posting to personal, institutional or third party websites are prohibited.

In most cases authors are permitted to post their version of the article (e.g. in Word or Tex form) to their personal website or institutional repository. Authors requiring further information regarding Elsevier's archiving and manuscript policies are encouraged to visit:

http://www.elsevier.com/authorsrights 


\title{
The influence of recent major crater impacts on the surrounding surfaces of (21) Lutetia
}

\author{
M. Jutzi ${ }^{\text {a }}$, N. Thomas ${ }^{\mathrm{a}, *}$, W. Benz ${ }^{\text {a }}$, M.R. El Maarry ${ }^{\text {a }}$, L. Jorda ${ }^{\text {b }}$, E. Kührt ${ }^{c}$, F. Preusker $^{c}$ \\ a Physikalisches Institut, University of Bern, Sidlerstr. 5, CH-3012 Bern, Switzerland \\ ${ }^{\mathrm{b}}$ Laboratoire d'Astrophysique de Marseille, 38 rue Frédéric Joliot-Curie, 13388 Marseille, France \\ ${ }^{\mathrm{c}} \mathrm{DLR}$, Institute of Planetary Research, Rutherfordstr. 4, Berlin-Adlershof, Germany
}

\section{A R T I C L E I N F O}

\section{Article history:}

Received 7 January 2013

Revised 4 April 2013

Accepted 14 May 2013

Available online 2 June 2013

\section{Keyword:}

Asteroids, surfaces

Impact processes

Cratering

Collisional physics

\begin{abstract}
A B S T R A C T
We present 3-D simulations of impacts into Asteroid 21 Lutetia, the subject of a fly-by by the European Space Agency's Rosetta mission to Comet 67P/Churyumov-Gerasimenko. Using a 3-D shape model of the asteroid, impacts of sizes sufficient to reproduce the observed craters in Lutetia's North Polar Crater Cluster (NPCC) as observed by the OSIRIS experiment have been simulated using the Smoothed Particle Hydrodynamics technique. The asteroid itself has been modelled both as a homogeneous body and as a body with an iron core.

Crater erasure in the vicinity of the NPCC has been observed by OSIRIS. The results show that this erasure has most probably been caused by ejecta deposition following the impact of a $2.3 \mathrm{~km}$ diameter projectile impacting at a velocity of $5 \mathrm{~km} \mathrm{~s}^{-1}$ (or an impact with similar energy). This would produce a crater of roughly $34 \mathrm{~km}$ in diameter comparable to the largest (and oldest) member of the NPCC. Erasure of craters via the shock associated with such an impact is shown to be less significant and does not reproduce the observed spatial distribution of erased craters or "ghost" craters.

Time series of the surface velocity fields resulting from the simulated impacts are also presented. It is suggested that the surface velocity field and velocity shear may play a role in the generation of lineaments. Our model calculations show that the velocity field lines around $50 \mathrm{~s}$ after impact exhibit a reasonable qualitative correlation with the orientation of lineaments observed on the entire visible surface of Lutetia. It is also shown that incorporation of a core of $25-30 \mathrm{~km}$ in diameter does not modify the velocity field evolution with time and, as such, the presence or otherwise of such a core cannot be inferred from lineament observations if this concept for their formation is valid.
\end{abstract}

(c) 2013 Elsevier Inc. All rights reserved.

\section{Introduction}

The European Space Agency's Rosetta spacecraft completed a flyby of the main-belt Asteroid (21) Lutetia on 10 July 2010 at a heliocentric distance of $2.72 \mathrm{AU}$ (Schulz et al., 2012). Lutetia is in the inner main-belt with an orbital semi-major axis of $2.43 \mathrm{AU}$ and an eccentricity of 0.1634 (Keihm et al., 2012). It was originally classified as M-class although its spectral properties are now known to be unusual with some characteristics being more similar to chondritic material (Belskaya et al., 2010; Tosi et al., 2012).

The main imaging system on Rosetta, OSIRIS (Optical, Spectroscopic, and Infrared Remote Imaging System; Keller et al., 2007), acquired a remarkable series of images during the encounter at scales down to $60 \mathrm{~m} / \mathrm{px}$. The images have been used to re-construct the global shape models of Lutetia (Preusker et al., 2012; Jorda et al., 2012). These models show a highly irregular shape

\footnotetext{
* Corresponding author. Fax: +41316314405.

E-mail address: nicolas.thomas@space.unibe.ch (N. Thomas).
}

$\left((126 \pm 1) \times(103 \pm 1) \times(95 \pm 13) \mathrm{km}^{3}\right)$. Using the density derived with the support of the radio science instrument (Pätzold et al., $2007,2011)$, the magnitude of the surface gravitational acceleration can be calculated to be $\sim 0.05 \mathrm{~m} \mathrm{~s}^{-2}$ but varies strongly over the surface. The north pole (Carry et al., 2010) is located near a depression which has been produced by multiple impacts - the North Polar Crater Cluster or NPCC (Thomas et al., 2012). Its approximate position is indicated in Fig. 1 together with the prime meridian and the position of the $270^{\circ} \mathrm{E}$ longitude according to the IAU definition. As a consequence of the high obliquity of Lutetia $\left(96.35^{\circ}\right)$, the sub-solar point was at $47^{\circ} \mathrm{N}$ at the time of the fly-by (Keihm et al., 2012). Fig. 1 also illustrates the basic regions on Lutetia as defined in Massironi et al. (2012) and Thomas et al. (2012). The reader should note that having a copy of Thomas et al. (2012) available to refer to while reading this paper will probably be necessary because of the frequent references to figures therein.

The relative surface ages of units on the visible surface have been discussed in Marchi et al. (2012), Massironi et al. (2012) and Thomas et al. (2012). The observations show that the NPCC 


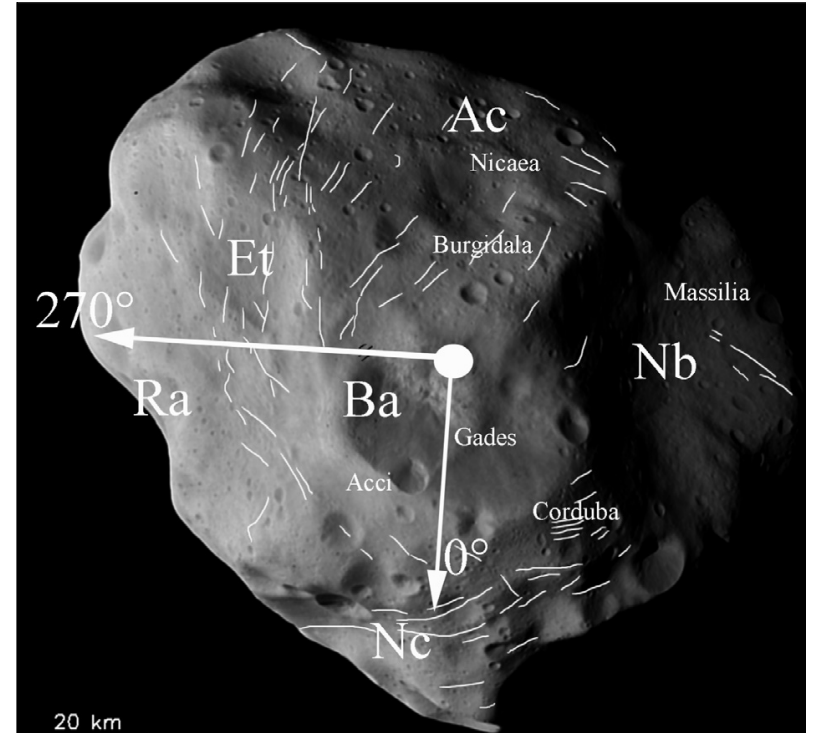

Fig. 1. Image NAC_2010-07-10T15.40.47.674Z_ID30_1251276001_F22 showing the prime meridian, the $270^{\circ} \mathrm{E}$ longitude, the main regions observed $(\mathrm{Ac}=$ Achaia, $\mathrm{Nb}=$ Narbonensis, $\mathrm{Nc}=$ Noricum, Et $=$ Etruria, $\mathrm{Ra}=$ Raetia, $\mathrm{Ba}=$ Baetica. Superimposed on the image are lines tracing a few of the visible lineaments. At high resolution, the lineaments are far more numerous, but those shown give a basic impression of their orientation.

must be geologically young. Estimates from the prevalence of boulders within and in the vicinity of the NPCC (Küppers et al., 2012) suggest an age of $<300 \mathrm{Ma}$. The crater statistics suggest even younger ages (<245 Ma; Marchi et al., 2012; their Fig. 8). The NPCC is however composed of several discrete impacts, four of which, named Corduba, Hispalis, Gades and Acci (see Thomas et al., 2012), define the appearance of the NPCC. While the $6 \mathrm{~km}$ Acci and $21 \mathrm{~km}$ Gades impacts (see Fig. 1) may have been the most recent, the Corduba $(34 \mathrm{~km})$ impact was obviously larger and also earlier as witnessed by both the later impact events and evidence of other, now buried, craters within its rim.

One of the most striking features of the OSIRIS images of Lutetia is the prevalence and diversity of lineaments on the surface. Some of the observed lineaments are more than $10 \mathrm{~km}$ long but they are very narrow and their depths are mostly below the resolution limit of the digital terrain model $(<\sim 100 \mathrm{~m})$. Lineaments have been observed on several other objects, most notably on the martian moon, Phobos (Thomas et al., 1979), 433 Eros (e.g. Buczkowski et al., 2008), 243 Ida (Sullivan et al., 1996; Asphaug et al., 1996) and 951 Gaspra (Veverka et al., 1994) but were not observed on 253 Mathilde (Veverka et al., 1997). Cassini observations indicate the presence of "grooves" on some small saturnian satellites (Morrison et al., 2009).

There is no accepted mechanism for producing these features on asteroids or small moons although some relationship to impact is widely assumed. In the case of Phobos, for example, the grooves and lineaments are assumed to be the result of the impact which produced Stickney crater (Thomas et al., 1979; Thomas and Veverka, 1979; Prockter et al., 2002) although an alternative view has been presented by Murray et al. (1994). Furthermore, the grooves of Pola Regio on 243 Ida have been linked to the impact that formed Vienna Regio (Asphaug et al., 1996). Hence, it is reasonable to suppose that the observed lineaments on Lutetia may have a similar impact-related origin.

The aim of this paper is two-fold. Firstly, can the relative absence of craters surrounding the NPCC (except for the Noricum region) be explained by an impact which formed one of the elements of the NPCC? Can the ejecta pattern be modelled and can parameters be selected which lead to a reproduction of the basic features of the ejecta blanket seen on Lutetia. In addition, can the more recent units within the Massilia crater be explained by the same phenomenon?

Secondly, can the orientations of lineaments, which are seen over most of the visible surface of Lutetia (Thomas et al., 2012), be a consequence of the same impact? Does the irregular shape of the body naturally lead to the different orientations of the lineaments seen in different regions? We use recent advances in Smoothed Particle Hydrodynamics (SPH) modelling to investigate this possibility.

In Section 2, we review the principal observations that we are seeking to model. In Section 3, we describe the model itself and the basic assumptions we use to construct and initialise the model runs. This is followed by a description of the cases run and the results obtained. This section is split into sub-sections describing the ejecta pattern, the propagation of the shock wave from an impact, and the velocity field. We shall see that many aspects of the observations can indeed be explained by a simple impact. The results also point to possible means of lineament production which we consider to be of major significance for future studies of small bodies.

\section{Pertinent observations}

Areas surrounding the NPCC appear to be covered (to a greater or lesser extent) with ejecta (Massironi et al., 2012; Thomas et al., 2012). The non-negligible gravitational attraction of Lutetia (Pätzold et al., 2011) implies an escape velocity of $\sim 70 \mathrm{~m} \mathrm{~s}^{-1}$. Hence, using simple scaling laws (see e.g. http://keith.aa.washington.edu/craterdata/scaling/index.htm; retrieved 18 February 2013 ), around $75-80 \%$ of material excavated in a hyper-velocity impact will fall back onto the surface.

The observed deposition blanket is not symmetric about the impact site - probably because of the irregular shape and gravity field of the object and, possibly, the angle of impact. The boundary between the NPCC (called Baetica in the unit definitions) and the Noricum region is very abrupt (see Thomas et al., 2012; Fig. 6) suggesting that little or no ejecta from any of the NPCC impacts exited in the direction towards $0-40^{\circ} \mathrm{E}$. The ejecta are most evident in the direction towards the oldest region, Achaia. In particular, Massironi et al. (2012) have illustrated that the ejecta from the NPCC partially covers the crater Burdigala. In Fig. 2, the area marked 'a' indicates this ejecta deposit although the exact definition of its margin is open to interpretation. The degree of burial of the older Achaia unit by this ejecta led to the inclusion of this region within the Baetica unit by Massironi et al.

As pointed out in Thomas et al. (2012), the Etruria region seems to be devoid of larger craters (Fig. 2). The appearance in general seems very different qualitatively from Achaia. Hence, some resurfacing by ejecta or other processes may well have occurred here too as part of one of the NPCC impact events.

The Nicaea depression forms a younger sub-unit within Achaia (Fig. 1). The structure is not of a regular crater and the depression has an unusual internal crater distribution (Thomas et al., 2012; Fig. 36). Massironi et al. (2012) infer from this that Nicaea is younger than the rest of the Achaia unit but that it is older than the oldest NPCC event. However, an alternative interpretation might be that, although Nicaea formed around the time of the Achaia unit or slightly later, it received a partial coating of ejecta, roughly conforming to the area marked out as region 'b' in Fig. 2, from the NPCC events. A similar explanation may apply to Burgidala (which is suggested to be of similar age; Massironi et al., 2012).

Thomas et al. (2012) noted that there were areas of different surface age within the Massilia crater in the Narbonensis region. 


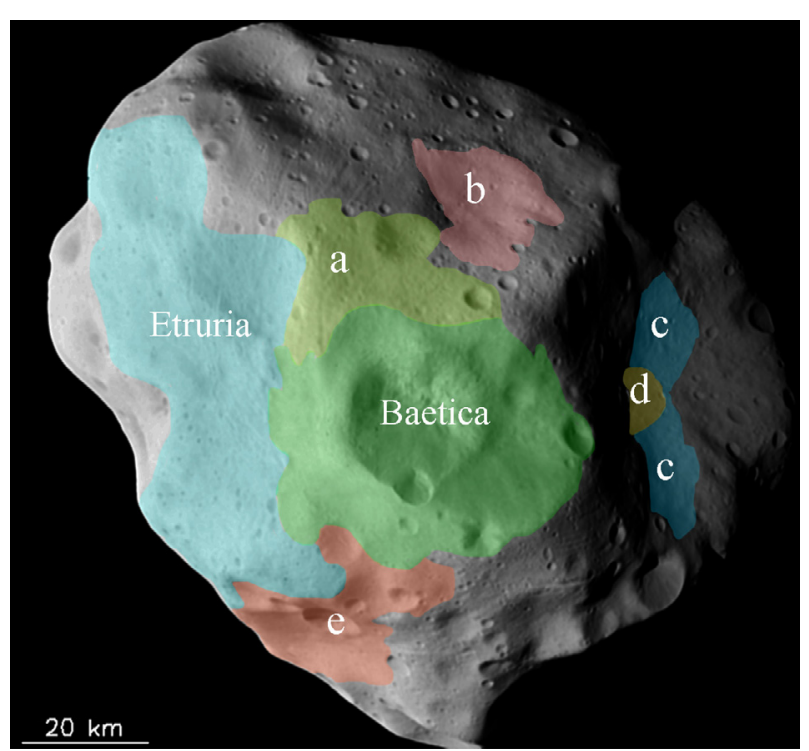

Fig. 2. Areas on Lutetia where there is evidence of ejecta deposition. The Baetica region has been influenced by the impacts in the NPCC. Region a shows relatively poor contrast consistent with a thin ejecta blanket. The Etruria region shows a dearth of larger impact crates. Regions b, c, d, and e also show a lack of impact craters as discussed in the text.

The regions marked ' $c$ ' in Fig. 2 were identified as possibly younger surfaces whilst Fig. 31 of Thomas et al. (2012) indicates a change of local slope which might be associated with a deposit covering at least the area marked 'd' in Fig. 2. This area may be larger than shown in Fig. 2 but shadowing and the oblique illumination on the rim of the Massilia depression makes this difficult to assess. We note that landslides (including slumping of the Sèquana ridge) down local gravitational slopes have been suggested as a possible explanation for these phenomena (Vincent et al., 2012). Below we suggest that ejecta from the NPCC may have been a substantial contributor to the re-surfacing.

The region marked ' $e$ ' in Fig. 2 corresponds to the youngest part of the Noricum region. There is a relative fresh but small impact crater near its centre. Adjacent craters appear older and covered with ejecta. The fresh impact may have been responsible for the ejecta but we will show below that ejecta from the NPCC might also have contributed prior to the youngest impact.

Lineaments are observed over the entire visible hemisphere of Lutetia except in the Baetica region (Thomas et al., 2012). There is a strongly preferred lineament orientation in each region but this orientation differs over the visible surface. In Fig. 1, the orientations of a few of the lineaments are indicated to give a global impression. Although, in the Noricum region, the lineaments roughly circumscribe the NPCC (see the "rose" diagram in Thomas et al., 2012) in other areas, notably Narbonensis, the preferred orientation is not circumpolar. In the Achaia region there is also evidence of criss-crossing of lineaments (see Thomas et al., 2012; Fig. 24). Different types, possibly of different age in some cases, could also be recognized. The alignment of lineaments can be compared with observations of Ida which showed no obvious arrangement (Sullivan et al., 1996).

\section{Model approach, initial conditions, and assumptions}

\section{1. $S P H$}

To perform the impact simulations we use a Smoothed Particle Hydrodynamics (SPH) code specially written to model geologic materials (Benz and Asphaug, 1994,1995; Jutzi et al., 2008). This code was validated by comparison with laboratory impact experiments involving solid and porous (Jutzi et al., 2009) materials. Using a newly implemented Drucker-Prager-like yield criterion, our method can also successfully reproduce the flow behaviour of fragmented (granular) material (Jutzi et al., 2013), which is important to study the late stages of crater formation. For the simulations presented in this paper, self-gravity is included and is computed using a grid-based gravity solver (Jutzi et al., 2013). The implementation is fully 3-D taking into account the shape of the asteroid (see below).

\subsection{Assumptions}

For the internal structure of the body we test three different models. In the first model, it is assumed that initially, the body is chemically and structurally homogeneous. The second model is based on the idea of a denser core resulting from (partial) differentiation (e.g. Weiss et al., 2012). In this model, the body consists of a core, a mantle and a porous crust. To study the effect of the core on the wave propagation, we also investigate a third model with a non-porous crust (i.e., in this model, mantle and crust are assumed to have the same properties - a two-layer model).

We note that incorporating a core requires many additional free parameters. In particular, the way it is implemented can have many consequences. For example, a strict finite boundary will provide reflections of shocks back towards the surface while gradual transitions may damp reflections and allow more energy to propagate through to the core. The inclusion of a core therefore opens up a huge range of parameter space which cannot be easily investigated within current computing capabilities. However, the cases investigated in this study provide an estimate of the importance of the effect of a core on the wave propagation.

To explain the abrupt transition between old and new surface units at the Baetica-Noricum boundary, we assume an impact coming in at an angle of $45^{\circ}$ with respect to the surface plane. This appears to reproduce the observations well (see below) but there may be alternative ideas to explain the origin of this boundary. We have also not investigated the range of angles which might provide similar results because of the excessive computational time required.

We assume that the most recent NPCC impacts have been responsible for the observed lineaments as well the obvious ejecta about the NPCC. For the lineaments, this is not a trivial assumption. However, the lineaments are, in general, not major constructs and are subject to erosion via space-weathering effects. Küppers et al. (2012) suggest that $200 \mathrm{~m}$ boulders would be eroded in timescales of a few hundred million years. Thomas et al. (2012) show that even the most significant lineaments are only 100-200 m deep. Hence, they do have to be fairly young to have avoided obliteration and their relative youth may explain why we have such a wonderfully preserved set of lineaments on Lutetia.

\subsection{3-D description of Lutetia}

Our numerical model of the shape of Lutetia has been retrieved from the images using the stereophotoclinometry technique (Gaskell et al., 2008) combined with stereo control points and shape from silhouette (Jorda et al., 2011). This technique has been applied before to retrieve the shape of the Asteroid (2867) Steins after the Rosetta flyby (Jorda et al., 2012). The shape model produced a body of $(126 \pm 1) \times(103 \pm 1) \times(95 \pm 13) \mathrm{km}^{3}$ and has a resolution of $\sim 100 \mathrm{~m}$, which is much higher than the spatial resolution in our SPH impact simulations. Therefore, only every 10th of the data points of the shape model is used to set up the 3D SPH model of Lutetia. For the simulations presented here, $\sim 5 \times 10^{6}$ 
SPH particles are used, which results in a spatial resolution of $\sim 500 \mathrm{~m}$. The resulting computing time is typically 20 days using 32 cores in parallel.

We have used the current shape model of Lutetia but have numerically filled-in the NPCC to simulate the pre-impact shape. The new surface has been created using the function

$f=\left(\left(1-\left(\frac{r_{x y}}{r_{0}}\right)^{a}\right)^{b}-1\right) d_{h}+d_{0}$

where $r_{x y}^{2}=\left(x-x_{0}\right)^{2}+\left(y-y_{0}\right)^{2}, x_{0}=12.5 \mathrm{~km}, y_{0}=2.5 \mathrm{~km} \quad$ and $r_{0}=26 \mathrm{~km}, d_{h}=4 \mathrm{~km}, d_{0}=46 \mathrm{~km}, a=3$, and $b=1.5$. The new surface is then defined by $\left|r_{\text {new }}-r_{\text {offset }}\right|<f$ where $r_{\text {offset }}=\left(x_{0}, y_{0}, 0\right)$. The parameters chosen allow a smooth transition from the new surface to the current shape model. However, it should be clear that the exact pre-NPCC shape is unknown. In order to investigate the influence of the pre-impact shape on our results, we have also tested a different shape of the surface covering the NPCC region. For this, we used a different approach and defined

$f_{2}=d_{0}\left(1-\left(\frac{r_{x y}}{r_{0}}\right)^{c}\right)+\left|r_{\text {surf }}-r_{\text {offset }}\right|\left(\frac{r_{x y}}{r_{0}}\right)^{d}$

where $d_{0}=46 \mathrm{~km}, r_{0}=27.5 \mathrm{~km}, c=4$, and $d=3.2$. Here, $r_{\text {surf }}$ defines the original surface. The new surface is then defined by $\left|r_{\text {new }}-r_{\text {offset }}\right|$ $<f_{2}$. Note that $f$ and $f_{2}$ have a different functional form. The two different initial shapes are shown in Fig. 3. The direction of the impact with respect to the model is indicated in Fig. 4.

\subsection{Material properties}

In structure model 1, Lutetia is modelled as a homogeneous, non-porous body with a bulk density of $\rho=3.4 \mathrm{~g} / \mathrm{cm}^{3}$ (Pätzold et al., 2011). In model 2 , the body consists of an iron core of $25 \mathrm{~km}$ diameter, a mantle $\left(\rho=3.3 \mathrm{~g} / \mathrm{cm}^{3}\right)$ and a porous crust $\left(\rho=1.65 \mathrm{~g} / \mathrm{cm}^{3}\right)$ with a thickness of $2 \mathrm{~km}$. The resulting bulk density in this model is about the same as in model $1(\rho=3.37 \mathrm{~g} /$ $\mathrm{cm}^{3}$ ). Model 3 uses an iron core of $30 \mathrm{~km}$ (the largest possible within the mass constraint) and a mantle $\left(\rho=3.3 \mathrm{~g} / \mathrm{cm}^{3}\right)$ and a nonporous crust (same material as the mantle). The resulting bulk density $\rho=3.74 \mathrm{~g} / \mathrm{cm}^{3}$ is close to the upper limit of the value inferred from the observations (Pätzold et al., 2011).

In all models, the material is assumed to be damaged (i.e., it is shattered and has no tensile strength) at the time of the NPCC impacts, based on the assumption that the large, pre-NPCC impacts (e.g. that forming the Massilia structure) shattered most of Lutetia.

We have used the Tillotson Equation of State (EOS) with basalt parameters (e.g. Melosh, 1989). For the range of impact velocities $(2.5-5 \mathrm{~km} / \mathrm{s}$ ) considered in our study (velocity here is used in the

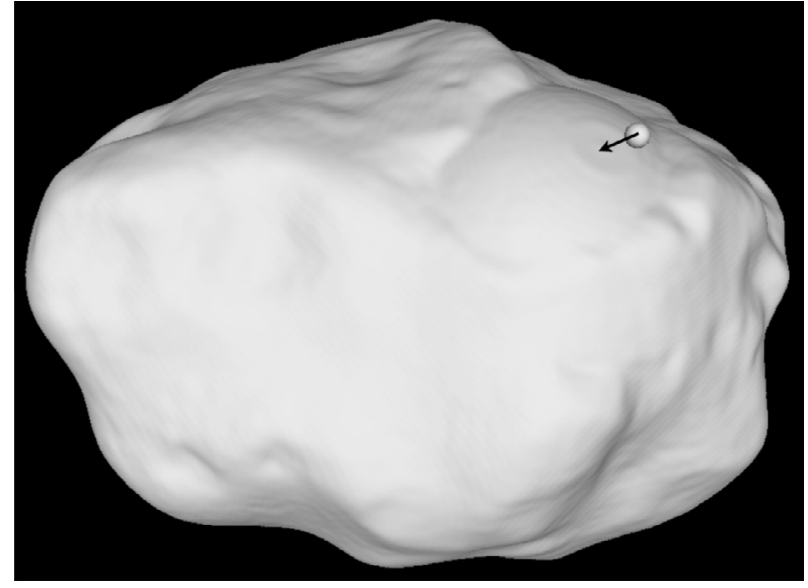

Fig. 4. The shape model of the asteroid used. The impactor is indicated and its direction of motion prior to the impact shown. Note the "filling in" of the NPCC.

sense of the magnitude of the velocity vector), no melting or vaporization is expected and the simple Tillotson EOS is appropriate. A yield stress of $0.5 \mathrm{GPa}$ and a coefficient of friction of 0.55 are used to model the rocky material. To model the porous crust, we use the porosity model described in Jutzi et al. (2008) with an initial distention of $\alpha_{0}=2$.

\section{Cases run and results}

Within the NPCC, Acci was the most recent event but at only $6 \mathrm{~km}$ in diameter, we suggest that this impact had little influence on the large scale surface structures observed with OSIRIS. The most recent of the larger impacts produced the $21 \mathrm{~km}$ diameter crater, Gades. The impact which produced the $34 \mathrm{~km}$ Corduba crater was obviously larger but older. It was not obvious which of these impacts could be responsible for the observed ejecta and lineaments and so we chose to investigate both possibilities. We assumed, in both cases, that there were no major impact structures at or close to the impact site. (This is a significant simplification for the $21 \mathrm{~km}$ impact model because the larger, $34 \mathrm{~km}$, impact structure had to have been produced previously. As will be seen below, however, the smaller impact appears to have been of insufficient energy to model the observations removing the need to study this in greater detail.)

We study the effect of impacts into the Baetica area on Lutetia in five different cases (see Table 1 ), where we consider different impact energies and internal structures. The first case, a, aims to
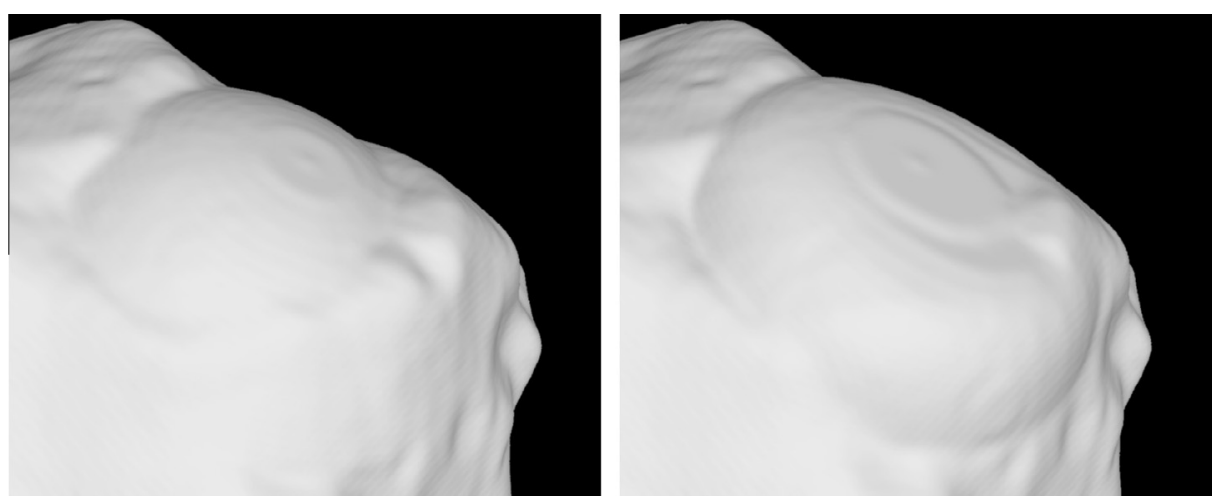

Fig. 3. The two models for the filling in of the NPCC simulating the appearance of the asteroid before the 34 km diameter impact. The model on the left is used as standard model for the simulations indicated in Table 1. 
Table 1

Input parameters for the impacts modelled.

\begin{tabular}{lllll}
\hline & $\begin{array}{l}\text { Projectile } \\
\text { diameter }(\mathrm{km})\end{array}$ & $\begin{array}{l}\text { Impact velocity } \\
(\mathrm{km} / \mathrm{s})\end{array}$ & $\begin{array}{l}\text { Impact } \\
\text { energy (J) }\end{array}$ & Structure \\
\hline (a) & 1.8 & 5 & $1.2 \mathrm{e} 20$ & $\begin{array}{l}\text { Model 1 } \\
\text { (homogeneous) } \\
\text { Model 1 } \\
\text { (homogeneous) } \\
\text { (b) } 2.3\end{array}$ \\
(c) 3.6 & 5 & $2.5 \mathrm{e} 20$ & $2.5 \mathrm{e} 20$ & $\begin{array}{l}\text { Model 1 } \\
\text { (homogeneous) } \\
\text { Model 2 (core/ } \\
\text { mantle/crust) } \\
\text { Model 3 (core/ } \\
\text { mantle) }\end{array}$ \\
(d) 2.3 & 5 & $2.5 \mathrm{e} 20$ & $2.5 \mathrm{e} 20$ & \\
(e) 2.3 & 5 & &
\end{tabular}

reproduce the smaller Gades crater whereas the initial conditions of cases b, c and e are chosen to reproduce the larger Corduba crater. In case d, the same impact energy is used, but because of the presence of the porous crust, the resulting crater dimension and the amount of ejecta are smaller.

For all simulations a-e, the standard filling shape (Fig. 3, left) is used. To investigate the influence of the pre-impact shape on our results, we perform one test run using the same initial conditions as in run b, but with a different filling shape (Fig. 3, right).

\subsection{Ejecta pattern}

Fig. 5 shows the deposition pattern of ejecta for three cases; models a, b, and d. The modelled ejecta blanket is colour-coded to give the thickness at each point on the visible hemisphere. This depth, $d h$, can be converted to a maximum diameter, $D_{\max }$, of craters that are covered and obscured by the ejecta. We use here

$D_{\max }=d h /(c * f)$

where $f$ is the depth/diameter ratio of the craters and $c$ is the minimum thickness (expressed as a fraction of crater depth) of the ejecta blanket which is necessary to cover the crater. Here we assume $c \sim 1 / 3$ and $f \sim 1 / 3$, resulting in $D_{\max } \sim 10 \mathrm{dh}$. A second scale has been introduced in Fig. 5 to show the result of this calculation.

It can be seen in case a that the ejecta does not extend far enough away from the NPCC to explain the observed ejecta deposit. It can also be seen however that ejecta seems to collect in local topographic lows. This is also seen in the other two cases. The stronger, higher energy, impacts (e.g. run b), which would lead to a $\sim 34 \mathrm{~km}$ diameter crater, give ejecta distributions which more closely match the observations. In particular, (1) the section of Achaia nearest Baetica is well covered by ejecta in the model, (2) Massironi et al. (2012) classified Nicaea within the Achaia region as a different unit on the basis of crater density and here it can be seen that deposition from the $34 \mathrm{~km}$ impact may have been responsible for this receiving more than $100 \mathrm{~m}$ depth of ejecta, (3) the Etruria region also receives a blanket sufficient to cover $1 \mathrm{~km}$ diameter craters which gives an explanation for Thomas et al.'s view that the region is depleted in intermediate-sized craters, (4) the Noricum region receives less ejecta which could thereby explain the observed sharp discontinuity in surface age between the Baetica and Noricum regions, (5) the Nr1d unit, which appears "fresh" and contains the best candidate for a recent impact crater (see Fig. 50 in Thomas et al., 2012) receives more than $200 \mathrm{~m}$ depth of ejecta which may contribute to its relatively fresh appearance, and (6) the northern face of the Massilia crater (Narbonensis) is

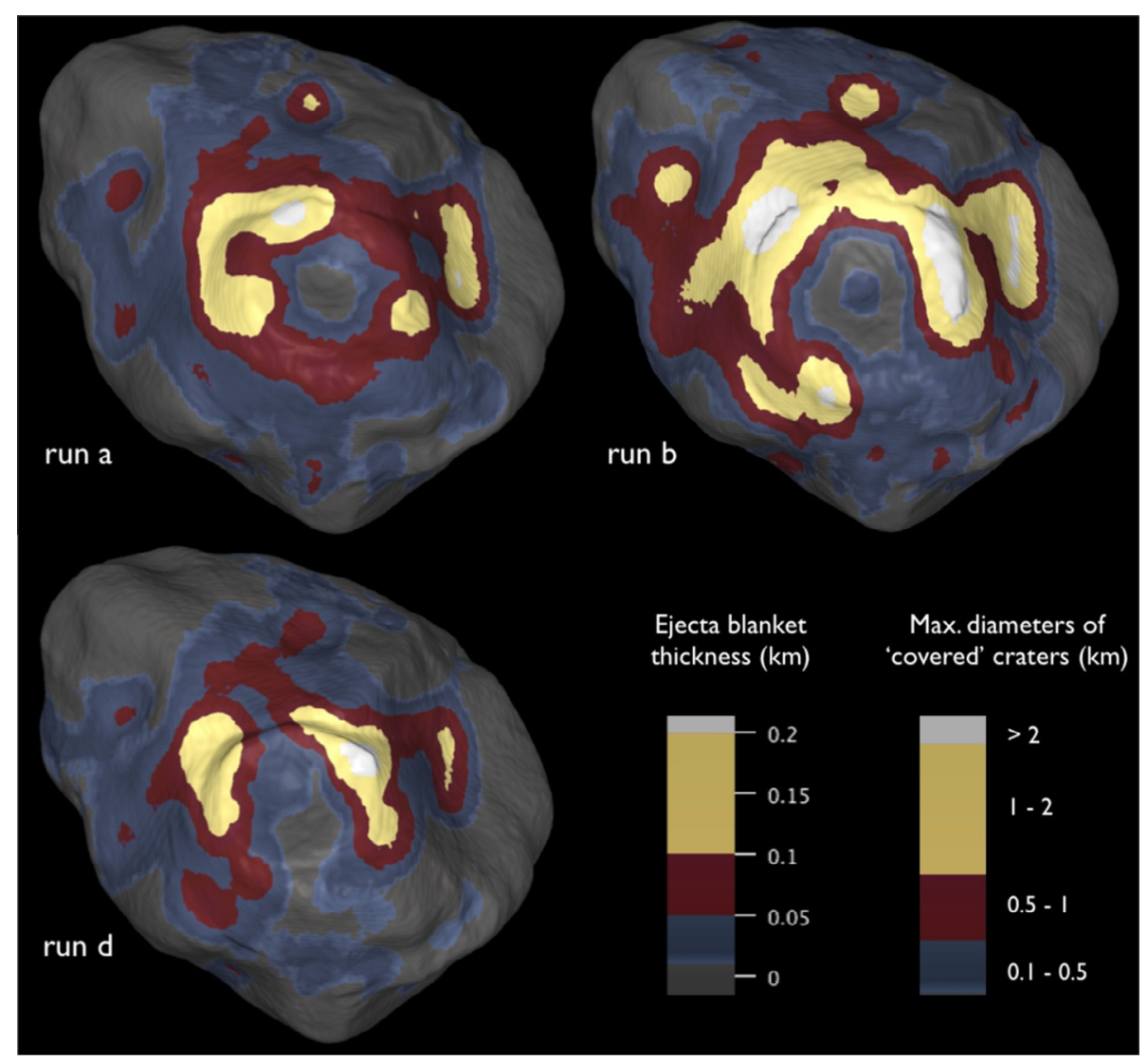

Fig. 5. Ejecta blanket thickness $d h$ and maximum size of craters covered by the ejecta. The colour code can be converted to a maximum diameter $D_{\text {max }}$ of the craters covered (see text) using the alternate colour bar. Shown are the cases a, b and d (see Table 1). 
covered with ejecta up to a thickness of $>200 \mathrm{~m}$. This could explain the younger units within Massilia (e.g. Nb1a and Nb1d in Fig. 3 in Thomas et al., 2012) and the change of slope seen within the crater (see Fig. 31 in Thomas et al., 2012).

Even though case $d$ uses the same impactor as case $b$, much less ejecta has been produced. This can be explained by the extra dissipation of energy in the porous crust (Jutzi et al., 2008). For such a structure, a more energetic impact would be needed to match more closely the observations. The differences between cases $b$ and c (not shown in Fig. 5) are very small. The ejecta distribution, a major computational driver, was not computed in case e.

Our comparison simulation (run b, but using the filling model of Fig. 3, right) indicates that a different pre-impact shape only affects some details of the ejecta distribution. Using a more flat pre-impact surface, the projectile produces slightly more ejecta. There is also more ejecta emplaced at the border of the crater because this region is initially higher. However, the overall pattern of the ejecta distribution does not change significantly.

\subsection{Seismic shock wave}

It has been suggested that the seismic shock wave resulting from impact is sufficient to erase craters. One might expect that this shaking would be irregularly distributed about the impact event because of the non-uniform shape of the object. However, in Fig. 6 we see that the irregular shape of the body seems to play a minor role. The figure shows the maximum velocity experienced by the surface during the impact event. This has been converted to the diameters of craters which would have been erased by this magnitude of shock. To compute the maximum diameters, $D_{\max }$, of craters erased by the shock wave, we follow Asphaug (2008) and assume that the velocity $v_{\text {crit }}$ necessary to degrade a structure of size $x$ is $v_{\text {crit }}=k(g x)^{0.5}$ where $g$ is the local gravitational acceleration and where we introduce a shape factor $k \sim 1 / 3$ which takes into account the geometry of the crater. This assumes the surface layer comprises loose material.

It can be seen that the disturbance of craters about the impact site is almost symmetric in all cases investigated and not consistent with the observed erasure of craters. The Noricum region would have suffered as much disturbance as the Achaia region. In addition, the diameters of the craters erased are somewhat smaller than that seen for the ejecta. Typically $500 \mathrm{~m}$ diameter craters would have been erased around the rim of the NPCC whereas $2 \mathrm{~km}$ craters would have been obscured by ejecta. Hence, erasure by the shock wave is unlikely to have been the dominant process.

\subsection{Velocity fields}

As discussed above, the resolution of our simulations is $\sim 500 \mathrm{~m}$ which is not enough to resolve and study directly the formation of lineaments. Nonetheless, we might hypothesize that their formation is the result of velocity shear as a consequence of impact. As the surface layer moves in response to the shock waves, one can envisage local differential motion occurring in regions with large medium-scale velocity gradients. This could result locally in extensional cracks or strike-slip faulting. This suggests that we should investigate the velocity field (as a function of time) to see if characteristic patterns arise in areas where lineaments are seen.

Fig. 7a shows snapshots of the velocity field at the surface of Lutetia in case a, up to $100 \mathrm{~s}$ after the impact event. Firstly, it can be seen that the initial sound wave takes $\sim 20 \mathrm{~s}$ to cross the body. Secondly, it is clear that the directions and magnitudes of velocity field lines change with time. Before the passage of the shock wave, there are no field lines because the velocities are zero (as also indi-

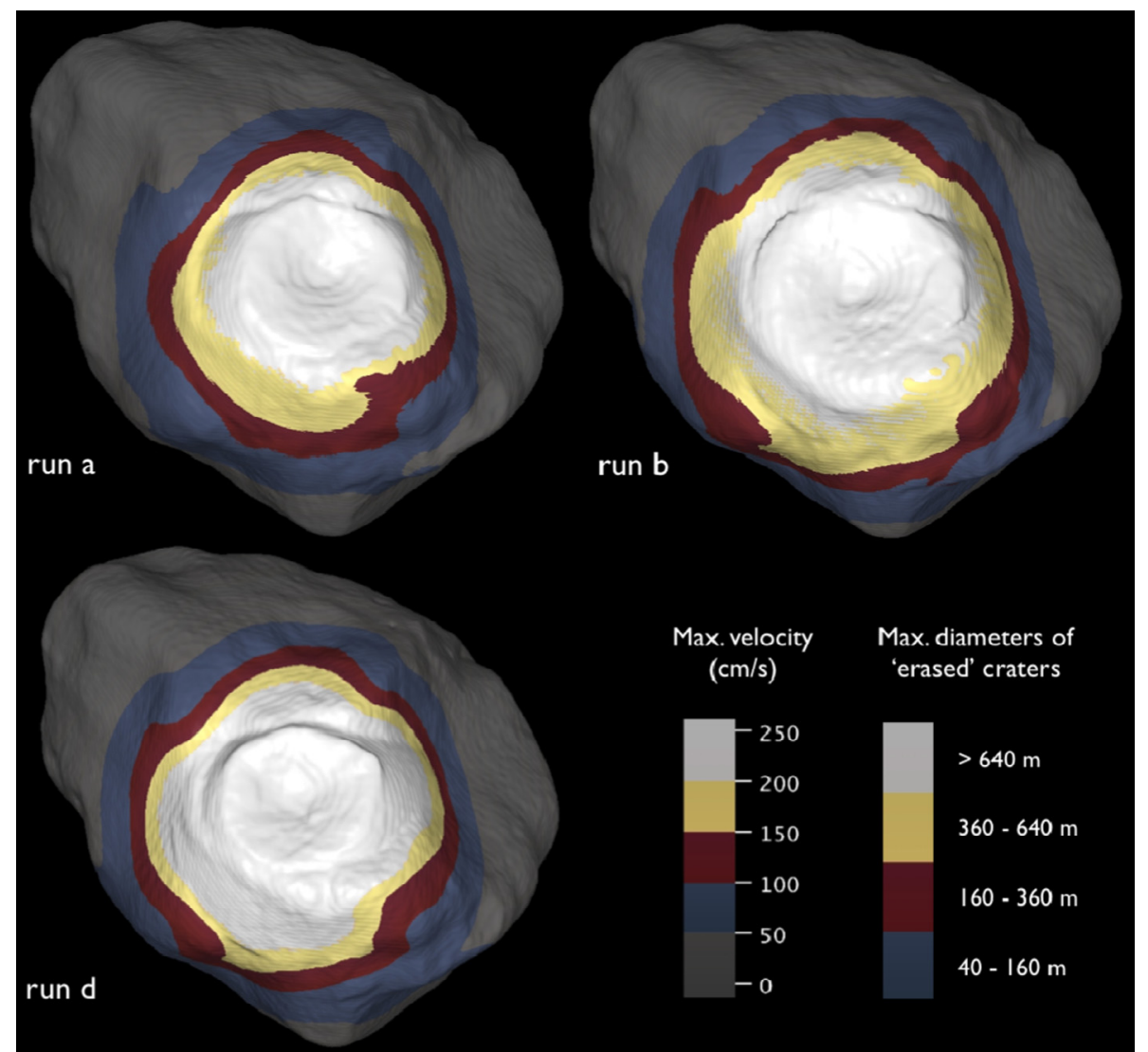

Fig. 6. Maximum velocity of the surface material induced by the shock wave produced by the impact. Shown are the results of the cases a, b and $d$ as described in Table 1 . 


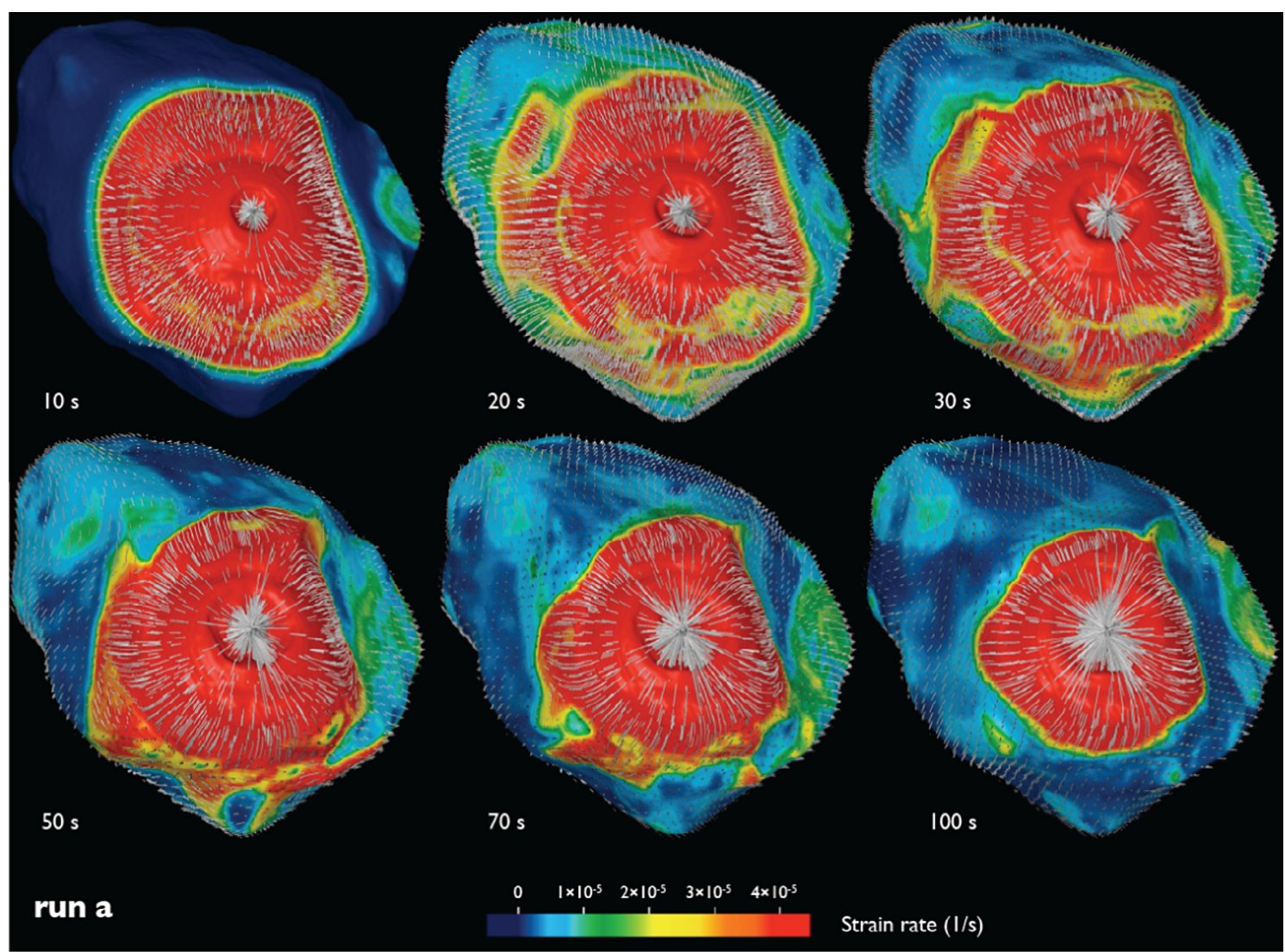

Fig. 7a. Time series of velocity field lines in case b. The 3D velocity field visualizes the velocity vectors of the material (SPH particles) at the actual time. It is overlapped by an iso-density surface. The mapped variable corresponds to the actual strain rate $(1 / \mathrm{s})$.

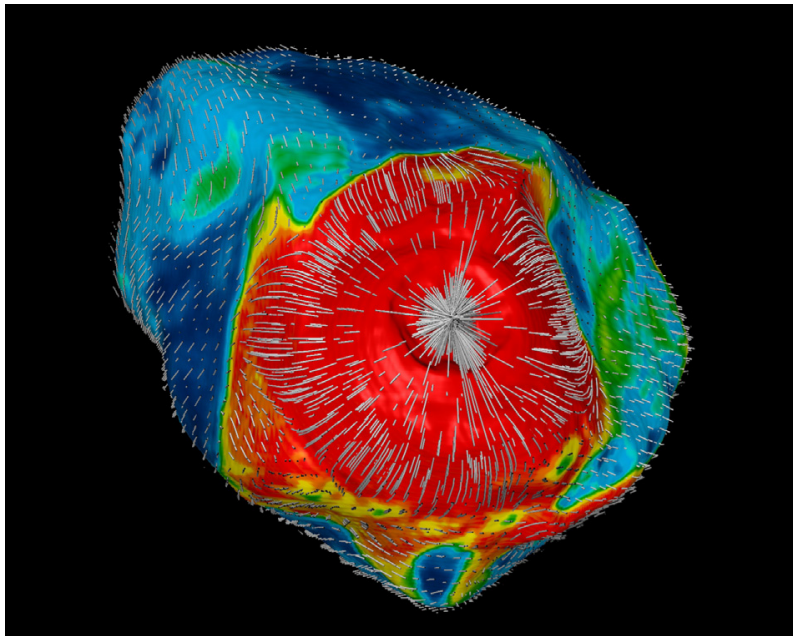

Fig. 7b. Case a at $t=50 \mathrm{~s}$.

cated by the strain rate, which is mapped in Fig. 7a). After the passage of the shock wave, the velocity field lines point away from the impact site in the area immediately surrounding the impact centre. In this region, the strain rates are higher $\left(>4 \times 10^{-5} 1 / \mathrm{s}\right)$ than further away from the impact point. After $\sim 30 \mathrm{~s}$, the region with the outward pointing velocity lines starts to get smaller, as the material flow slows down. Outside this region, the velocity field shows non-regular, and in some places divergent, patterns. $100 \mathrm{~s}$ after the impact, the strain rate is close to zero some distance away from the impact point, indicating that the velocities are very small (in this region, the field lines are mostly aligned) as the waves are damped.

Regarding the formation of cracks/lineaments, the field lines at the time $t=50 \mathrm{~s}$ show the most interesting pattern (see Fig. 7b). At this timestep, the velocity pattern correlates with some of the lineaments observed on Lutetia (Fig. 1).

The possible correlation between the velocity field and the lineaments is illustrated in more detail in Figs. 8-10. In Fig. 8, a section of an OSIRIS image (NAC_2010-07-10T15.41.35.529Z_ID30_1251276003_F22) is provided which shows the Noricum region. The data have been contrastenhanced (by unsharp masking). The middle panel identifies a number of lineaments in the image and shows their co-alignment in different sections of the image. The bottom panel shows the same region in the model. Note how the velocity vectors appear reasonably well aligned with the lineaments. Most of the lineaments are going from left to right (west-east) in this region and the model agrees fairly well with this. However, it should also be pointed out that, in the model, there are vectors which are indicating motion radially outward from the impact site (to the top of the image). There are some lineaments roughly co-aligned with this direction seen in the image (upper left; marked in orange) but these features are relatively weak.

The models all show that the radial motion away from the impact site is the most persistent feature of the velocity field. The lack of strong signatures (in the form of lineaments) of this motion might be an indication that fluidization and subsequent settling of the material occurred in the immediate vicinity of the impact. Shear forces were effectively zero before the material solidified. Further from the impact site the material remained more consolidated (less 

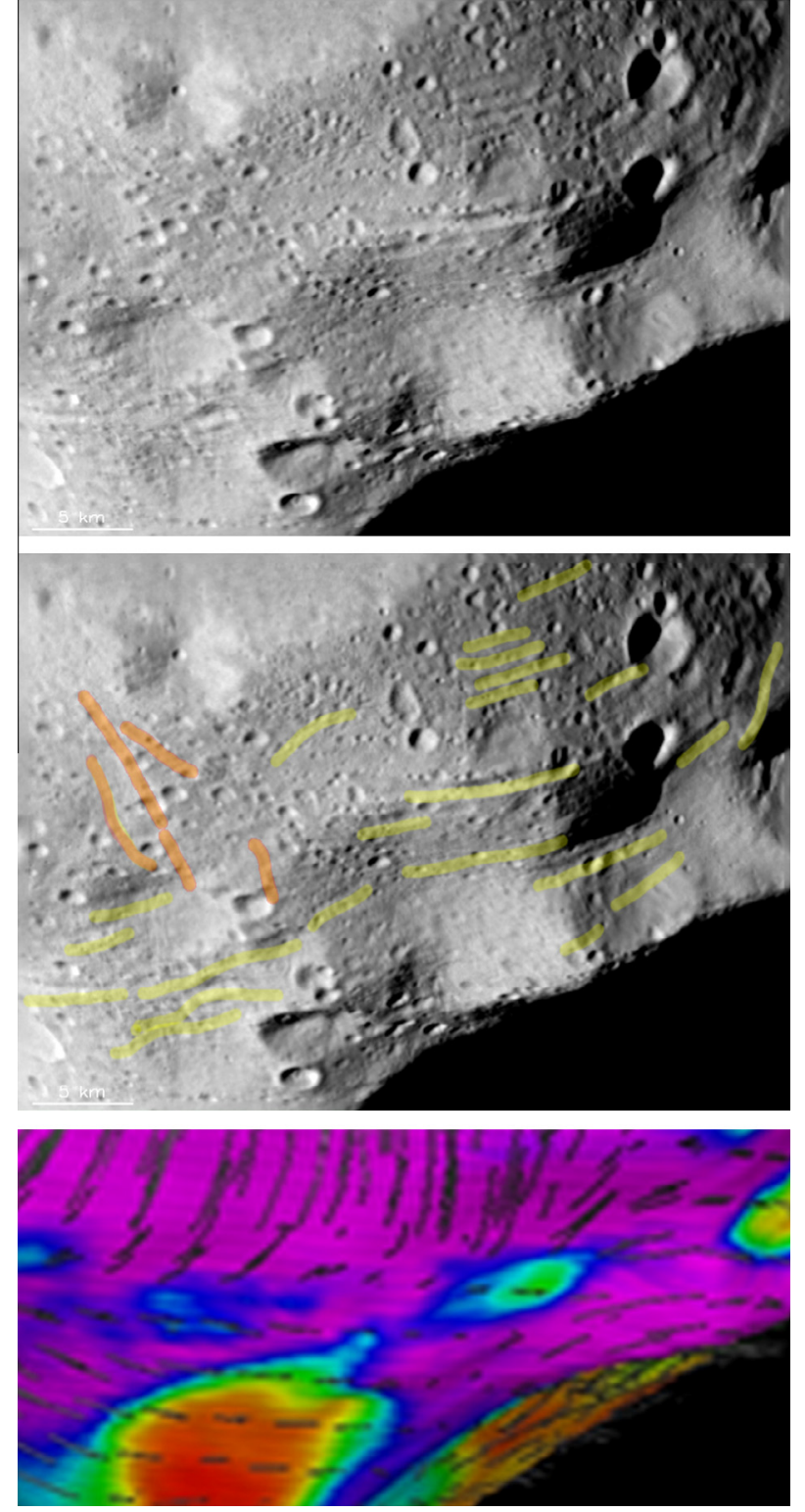

Fig. 8. Top: Part of image NAC_2010-07-10T15.41.35.529Z_ID30_1251276003_F22 acquired by OSIRIS during the fly-by of 21 Lutetia. The image section shows part of the Noricum region. Centre: Lineaments seen in the image have been emphasized and marked with different colours which are referred to in the text. Bottom: Modelled velocity vector field for this region $50 \mathrm{~s}$ after impact (case a). Note the (qualitative) correlation in the lineament direction (centre) with the velocity vectors (bottom).

fluid) so that shear motion could occur without settling and hence lineaments were preserved.

In Fig. 9, we see the boundary between the Narbonensis and Achaia regions which partially comprises the steep Sèquana ridge. As before, lineaments have been identified and overlaid on the image in the middle panel. The lineaments go in different directions. A lineament group (marked in yellow) at least $30 \mathrm{~km}$ long extends from the base of the Sèquana ridge into the Narbonensis region towards the bottom of the image section. Along the face of the ridge itself, there are lineaments which are almost orthogonal (bottom left; marked in orange). Other lineaments (centre right; marked in blue) have intermediate directions and there is an impression of a change in the orientation as one moves from left to right over the image section. The corresponding section of the velocity field
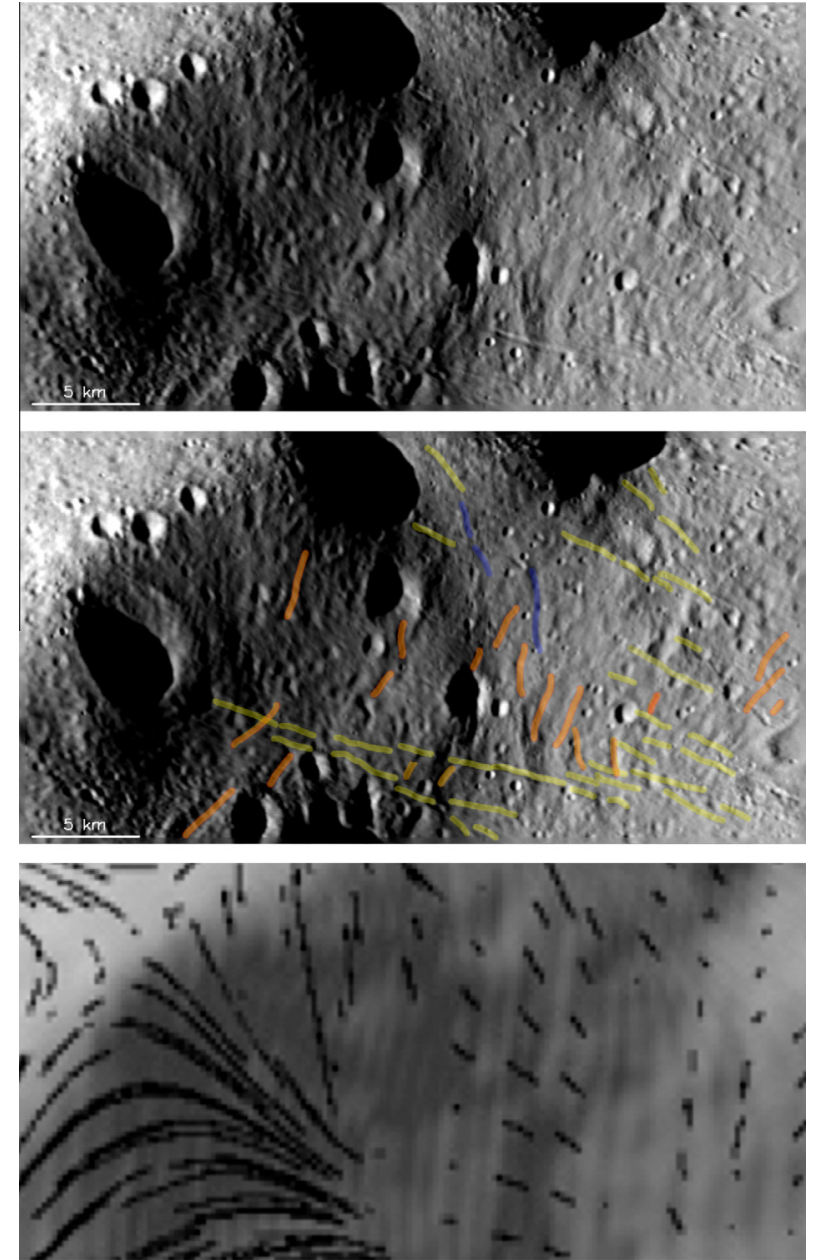

Fig. 9. Top: Part of image NAC_2010-07-10T15.42.47.523Z_ID30_1251276001_F22 acquired by OSIRIS during the fly-by of 21 Lutetia. The image section shows part of the Narbonenis region. Centre: Lineaments seen in the image have been emphasized and marked with different colours which are referred to in the text. Bottom: Modelled velocity vector field for this region $50 \mathrm{~s}$ after impact (case a). Note the changes in directions of the velocity vectors over the surface. (For interpretation of the references to colour in this figure legend, the reader is referred to the web version of this article.)

from the model calculation, $50 \mathrm{~s}$ after the impact, is shown in the lower panel. There are similarities between the vector orientations and the observed lineament orientations. Towards the bottom left, we see a sharp change in orientation of the velocity field which is seen in the lineaments. The orientation of the velocity vectors middle right agrees rather well with the lineament orientation. There is a change in the vector orientation in the centre of the section as one moves from left to right and the velocity vector orientation to the right is rather similar to that of the lineaments.

While this is visually quite convincing, there are disagreements. One sees, for example, lineaments along and at the base of the ridge which are in some cases orthogonal to that predicted by the model. Furthermore, there is no real correlation between the magnitude of the velocity vectors and the visual appearance of the lineaments. However, this may, in turn, be a problem of resolution.

A further example is given in Fig. 10 which is a section of the Etruria region. This area is particularly rich in lineaments. The main trend is from bottom to top of the image section but with some lineaments crossing each other at acute angles towards the top right. The lower panel again shows the velocity field of the model (rotated to simulate the viewing direction for the acquired image). 

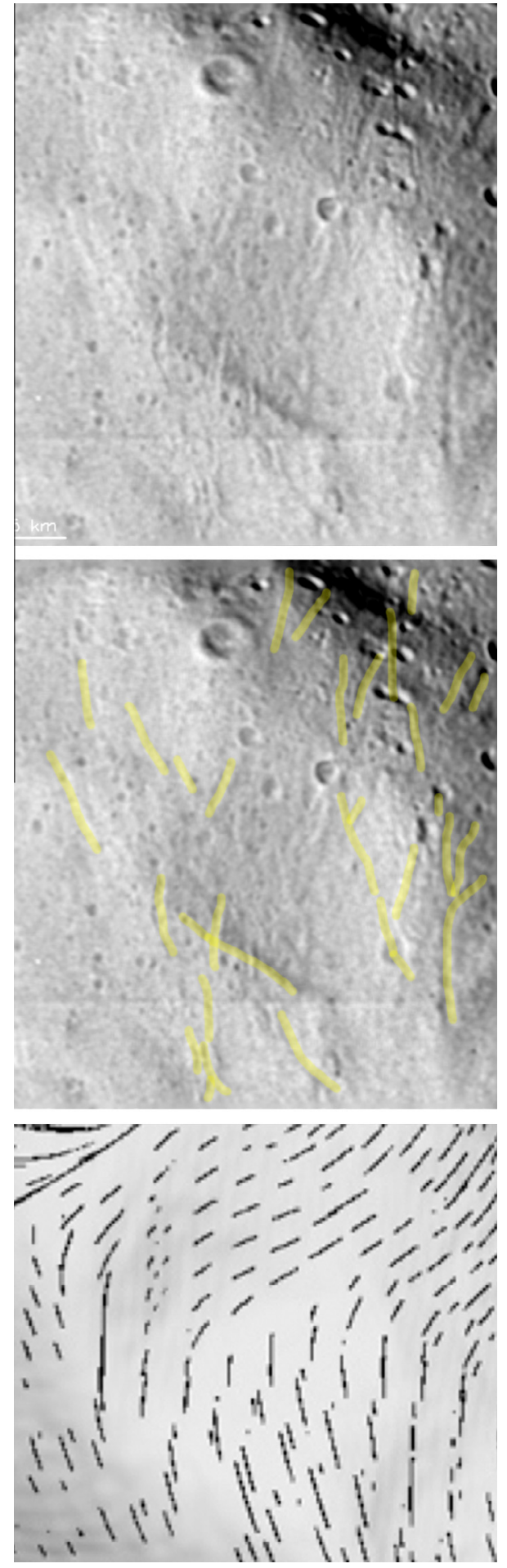

Fig. 10. Top: Part of image NAC_2010-07-10T15.38.15.051Z_ID30_1251276004_ F82 acquired by OSIRIS during the fly-by of 21 Lutetia. The image section shows part of the Etruria region. Centre: Lineaments seen in the image have been emphasized. Bottom: Modelled velocity vector field for this region $50 \mathrm{~s}$ after impact (case a). Note the changes in directions of the velocity vectors over the surface and the similarity of the pattern to the observed lineament pattern.

The trend (with lineaments oriented a few degrees from vertical) seen in the data to the bottom and left of the image is again seen in the model's velocity field. There are also changes in direction associated with crossing Rhenus Rupes (Thomas et al., 2012) into the depression feature at the centre of the image section. To the top right of the image section, there is evidence of segmented faults cross-cutting. In the model, this is an area where there seems to be relatively abrupt change in the orientation of the velocity field by around $45^{\circ}$. The co-alignment of the lineaments and the modelled velocity is not exact, however.

Figs. 8-10 are intriguing and suggest that there is a relationship between lineaments and the velocity field. However, the exact mechanism is completely unclear. In particular, the simple movement of the surface uniformly in one direction should not result in any modification of the surface except possibly some settling of loose material. To generate the extensional cracks seen in certain areas on Lutetia, divergence in the velocity field is needed while strike-slip motion requires shear in the velocity field. When viewing the details of the velocity field in the model, there are areas of divergence but we also see lineaments where the motion is roughly uniform in one direction. In the latter case, local inhomogeneity (in mass or physical structure) might provide a mechanism such that the acceleration of the surface layer differs locally. The surface layer of our model here is uniform.

In Fig. 11, the velocity field lines at a certain point in time (50 s) for the runs a-e are compared. As it can be seen, the cases b, $c$ and $e$ are very similar. These runs have the same impact energy. These results therefore indicate that for a given impact energy, the induced velocity field (i) does not (strongly) depend on the projectile size and (ii) is not significantly affected by a core $<30 \mathrm{~km}$ (i.e., the wave reflected back at the core-mantle boundary has been significantly weakened before it reaches the surface). However, a porous crust of $2 \mathrm{~km}$ thickness does affect the wave propagation significantly (case d). Although this case has the same impact energy as the cases b, c and e, the velocity field and the strain rate map at $50 \mathrm{~s}$ are more similar to the case a with the lower impact energy (case a). As discussed in Section 4.1, the dissipation of energy in the porous crust leads to a different behaviour (in this case, the damping of the shock wave). We conclude from this that this type of study cannot be used to constrain the size of any putative core but that the porosity of the uppermost layer of the asteroid provides a damping effect.

\section{Discussion and implications}

The model shows that craters up to several kilometres in diameter could have been buried by ejecta from the impacts which formed the North Polar Crater Cluster. The ejecta were inhomogeneously deposited about the impact site with accumulations in low-lying terrain. This appears to agree qualitatively with the observations from the imaging system on Rosetta. For the selected parameters, shock erasure of craters does not reproduce the observations because the erasure should have been more symmetric about the impact site and seems to be a less effective mechanism, i.e. the maximum diameter of erased crater at a specific distance from the impact site is considerably less for the shock erasure mechanism than for the ejecta deposition mechanism. There is no obvious way to reverse this conclusion without requiring highly unusual and non-justifiable initial conditions (e.g. a strongly inhomogeneous distribution of shock damping within the target body).

The observed lineaments on Lutetia neither perfectly circumscribe the NPCC nor do they radiate away from it. Because of their shallowness and the repeated bombardment by dust and meteorites, we assume that they are relatively young and related to the NPCC impact(s). Their youth and that they have been created by the NPCC impacts are, however, both assumptions which cannot be directly proven. If true, however, the model shows that as the acoustic waves pass through the asteroid, the velocity of the surface motion changes. The non-uniform shape of the asteroid plays a major role in modifying the orientation of the surface velocity 


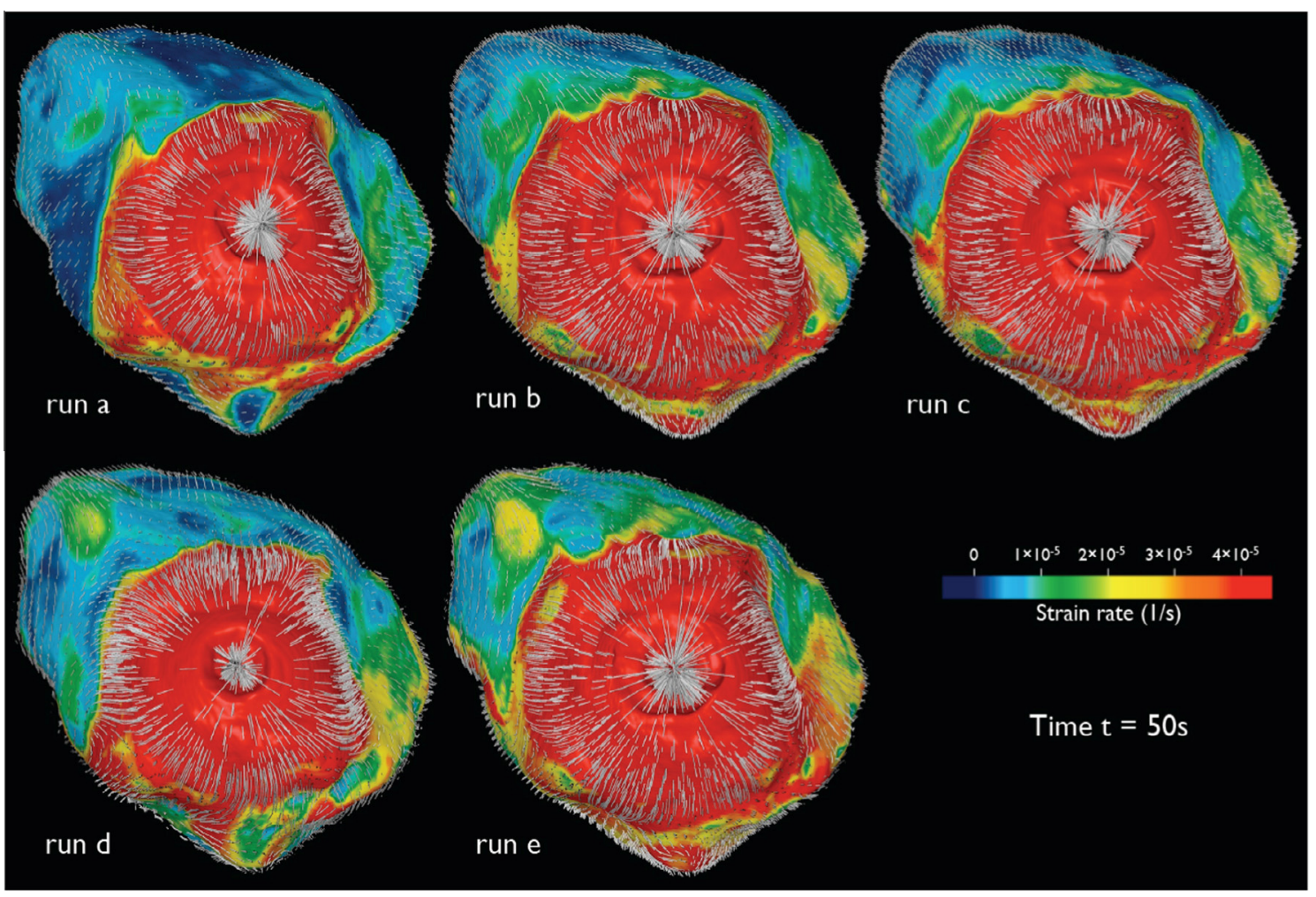

Fig. 11. Same as Fig. 5a but for cases a-e at a fixed time step $t=50 \mathrm{~s}$.

vectors with time and, hence, studies of the effects of impacts into small bodies must include the 3-D shape. The speed of the initial wave crossing the body is around $3 \mathrm{~km} / \mathrm{s}$ and arises from the choice of material. (It should be noted that the estimated speed of sound in regolith is typically between 100 and $300 \mathrm{~m} / \mathrm{s}$. However, the depth of the regolith is likely to be small compared to the resolution of the model and is therefore probably not applicable.) As the shock waves travel through the medium and are reflected from boundaries, the velocity vectors are slowed and deviate from radial in response to the 3-D shape. At certain times, the velocity vectors align with the orientations of the observed lineaments even if these are not radial or circularly symmetric about the impact site. Hence, we seem to have a relationship which could be used to explain the creation and orientations of the lineaments simultaneously through one isolated event. On the other hand, the orientations of the velocity vectors at the surface change with time as the event proceeds.

We have isolated one moment during the evolution of the impact (50 s after initial impact) and shown that at this time (2.5x the time needed for the initial shock wave to cross the body) there is a qualitative correlation between the surface velocity vectors and the orientation of lineaments in several places on the body where lineaments in several orientations are seen. However, there is no obvious physical reason why the velocity field should be important specifically at this time or indeed why the lineaments should be aligned to the velocity vectors. One can speculate that shear or divergence with respect the main direction of the motion is responsible for generating the lineaments but this will be extremely difficult to prove unless models are generated with $>10$ times higher resolution - something which is currently computationally impossible - at least for Lutetia. However, one can assign a characteristic time $\left(t_{\text {flow }}\right)$ to the material flow by taking the typical surface velocity and dividing by the surface gravity, i.e. $v / g$. On Lutetia, with surface velocities of $1-2 \mathrm{~m} / \mathrm{s}$ and $g \sim 0.05 \mathrm{~m} \mathrm{~s}^{-2}$, we obtain $t_{\text {flow }}=20-40 \mathrm{~s}$ which suggests a relationship between this and the time at which the velocity field shows most similarity to the lineament orientations.

There do remain inconsistencies in our interpretation with velocity vectors not being aligned with lineaments. One can have various modes of fracturing that orient themselves differently to a given stress field depending on whether they are compressive, tensile, or shear fractures. This will largely depend on the "local stress field", the subsurface microstructure and its physical strength which is mostly neglected here. So for given parts of the surface with certain geometries or internal structure, the local stress fields may overcome different material thresholds and create fractures with orientations that do not show the same reaction towards the velocity field. Unfortunately, this is essentially introducing additional free parameters for which we have few or no constraints.

A further potential difficulty is that, by using simple calculations, it can be shown that flight times for ejecta are typically an order of magnitude longer than the time for the velocity field to reach the required orientation $(50 \mathrm{~s})$. Hence, one might expect the lineaments created by the velocity field to have been buried by the ejecta in many areas. The presence of lineaments in the Etruria region is therefore not entirely consistent with the presented model.

The lineaments seen on the martian moon, Phobos, are widely regarded as having been produced by the impact which formed the crater Stickney (Thomas et al., 1979, 1992). A key difference between the lineaments on Lutetia and those on Phobos is that the lineaments on Phobos mostly radiate from the Stickney impact site whereas on Lutetia the lineaments mostly circumscribe the NPCC. In addition, lineaments can be seen on the rim of Stickney (see, for example, Thomas et al., 2010) whereas on Lutetia the crater rims show little evidence of lineaments.

The scales (energies) of the two impacts creating Stickney and the NPCC were vastly different but, if the interpretation of lineaments being roughly parallel to the local velocity has some validity, 
the question then becomes why Stickney has retained the signature of the velocity field close to the impact while Lutetia has lost it. Some relationship to the magnitude of the impact and the degree and duration of fluidization of the crater rim might be conceivable. It is our intention to investigate this further by modelling the Stickney impact using the same code.

Although the relationship of lineaments to the velocity field/ shear remains far from proven here, this work shows that we are approaching a point where meaningful constraints on lineament formation can be described which may in turn constrain the physical properties of the impacted medium.

Finally, one clear result of this study is how little effect the inclusion of a dense core has on the impact related phenomena. While it is not new that the formation of the crater is unaffected by a core that is appreciably deeper than the final crater depth, the fact that reflected energy from such a core also fails to generate obvious differences in the patterns of the impact-induced velocity field at the surface is perhaps surprising and novel. This should be compared with the case of Vesta. Here, the specific energy (kinetic energy of the projectile/target mass) of the considered impacts (Buczkowski et al., 2012; Bowling et al., 2013) is approximately a factor of 100 higher than for the impacts on Lutetia studied here. For these large impacts on Vesta, the depth of the transient crater almost reaches the core/mantle boundary. As a result, the core can significantly affect propagation of the shock wave produced by the impact.

The relative low energy impacts on Lutetia studied here produce much smaller craters (with respect to the size of the body and the depth of the core/mantle boundary). As our results indicate, in this case the core plays only a little role in modifying the velocity field evolution around the impact crater.

\section{Conclusions}

The ejecta distribution on 21 Lutetia resulting from the NPCC impacts is highly inhomogeneously distributed. This deposition pattern can be roughly reconstructed by using a 3D Smoothed Particle Hydrodynamics (SPH) model with a $2.5 \times 10^{20} \mathrm{~J}$ impact at $45^{\circ}$ with respect to the surface normal. This geometry seems to provide a plausible explanation for the sharp boundary between the younger Baetica region and the neighbouring (older) part of the Noricum region. Burial by ejecta of $2 \mathrm{~km}$ diameter craters close to the impact site is achieved with the model.

The size of the impact required to produce the deposition pattern is more consistent with the production of the $34 \mathrm{~km}$ diameter Corduba crater structure. The smaller impact which produced the Gades impact structure could have produced a similar pattern but the depth of the ejecta seems to be insufficient.

The ejecta deposition pattern in the model suggests that re-surfacing of some areas in the Massilia crater by ejecta could have occurred. The deposition pattern in our preferred model is qualitatively consistent with observed younger units in both Narbonensis and the Noricum unit, Nr1d (Thomas et al., 2012).

Crater erasure by the shock waves generated by the impacts is more symmetric about the impact site and does not reproduce the observed spatial distribution of erased craters. It is also, on the whole, a less effective process for crater erasure.

The origin of the lineaments remains difficult to assess because the model still has insufficient resolution. On the other hand, time series of the surface velocity fields resulting from the simulated impacts have been presented. Our model calculations show that the velocity field lines around $50 \mathrm{~s}$ after impact exhibit a reasonable qualitative correlation with the orientation of lineaments observed on the most of visible surface of Lutetia. This suggests that the surface velocity field and velocity shear may play a role in the generation of lineaments although there are numerous uncertainties in the mechanism. The interior structure (core as opposed to homogeneous) of the body plays little or no role in modifying the velocity field evolution with time for reasonable choices of core size.

The results here show that we may have tools to investigate the production mechanism of structures which are ubiquitous on small bodies in the Solar System.

\section{Acknowledgments}

We acknowledge support from the Swiss National Science Foundation through Grant 200020_140579 and through the SNSF Ambizione programme. We also thank the two referees for their excellent comments and suggestions.

\section{References}

Asphaug, E., 2008. Critical crater diameter and asteroid impact seismology. Meteorit. Planet. Sci. 43, 1075-1084.

Asphaug, E., Moore, J.M., Morrison, D., Benz, W., Nolan, M.C., Sullivan, R.J., 1996. Mechanical and geological effects of impact cratering on Ida. Icarus 120, 158184

Belskaya, I.N. et al., 2010. Puzzling Asteroid 21 Lutetia: Our knowledge prior to the Rosetta fly-by. Astron. Astrophys. 515, A29. http://dx.doi.org/10.1051/ 0004-6361/201013994.

Benz, W., Asphaug, E., 1994. Impact simulations with fracture. I - Method and tests. Icarus 107, 98-116.

Benz, W., Asphaug, E., 1995. Simulations of brittle solids using smooth particle hydrodynamics. Comput. Phys. Commun. 87, 253-265.

Bowling, T.J., Johnson, B.C., Melosh, H.J., 2013. Formation of Equatorial Graben Following the Rheasilvia Impact on Asteroid 4 Vesta. LPI Contributions, 1719, 1673.

Buczkowski, D.L., Barnouin-Jha, O.S., Prockter, L.M., 2008. 433 Eros lineaments: Global mapping and analysis. Icarus 193, 39-52.

Buczkowski, D.L. et al., 2012. Large-scale troughs on Vesta: A signature of planetary tectonics. Geophys. Res. Lett. 39, L18205.

Carry, B. et al., 2010. Physical properties of the ESA Rosetta target Asteroid (21) Lutetia. II. Shape and flyby geometry. Astron. Astrophys. 523, A94.

Gaskell, R.W. et al., 2008. Characterizing and navigating small bodies with imaging data. Meteorit. Planet. Sci. 43, 1049-1061.

Jorda, L. et al., 2011. Shape and physical properties of Asteroid (21) Lutetia from OSIRIS images. In: EPSC-DPS Joint Meeting 2011, held 2-7 October 2011 in Nantes, France, p. 776.

Jorda, L., Lamy, P., Gaskell, R., Kaasalainen, M., Groussin, O., Besse, S., 2012. Asteroid (2867) Steins: Shape, topography and global physical properties from OSIRIS observations. Icarus 221, 1089-1100.

Jutzi, M., Benz, W., Michel, P., 2008. Numerical simulations of impacts involving porous bodies. I. Implementing sub-resolution porosity in a 3D SPH hydrocode. Icarus 198, 242-255.

Jutzi, M., Michel, P., Hiraoka, K., Nakamura, A.M., Benz, W., 2009. Numerical simulations of impacts involving porous bodies. II. Comparison with laboratory experiments. Icarus 201, 802-813.

Jutzi, M., Asphaug, E., Gillet, P., Barrat, J.-A., Benz, W., 2013. The structure of the Asteroid 4 Vesta as revealed by models of planet-scale collisions. Nature 494, 207-210.

Keihm, S., Tosi, F., Kamp, L., Capaccioni, F., Gulkis, S., Grassi, D., Hofstadter, M., Filacchione, G., Lee, S., Giuppi, S., Janssen, M., Capria, M., 2012. Interpretation of combined infrared, submillimeter, and millimeter thermal flux data obtained during the Rosetta fly-by of Asteroid (21) Lutetia. Icarus 221, 395-404.

Keller, H.U. et al., 2007. OSIRIS - The scientific camera system onboard Rosetta. Space Sci. Rev. 128, 433-506.

Küppers, M. et al., 2012. Boulders on Lutetia. Planet. Space Sci. 66, 71-78. http:// dx.doi.org/10.1016/j.pss.2011.11.004

Marchi, S. et al., 2012. The cratering history of Asteroid (21) Lutetia. Planet. Space Sci. 66, 87-95. http://dx.doi.org/10.1016/j.pss.2011.10.010.

Massironi, M. et al., 2012. Geological map and stratigraphy of Asteroid 21 Lutetia. Planet. Space Sci. 66, 125-136.

Melosh, H.J., 1989. Impact Cratering: A Geologic Process. Oxford University Press, Oxford, UK.

Morrison, S.J., Thomas, P.C., Tiscareno, M.S., Burns, J.A., Veverka, J., 2009. Grooves on small saturnian satellites and other objects: Characteristics and significance. Icarus 204, 262-270.

Murray, J.B. et al., 1994. The origin of Phobos' grooves and crater chains. Planet. Space Sci. 42, 519-526.

Pätzold, M. et al., 2007. Rosetta Radio Science Investigations (RSI). Space Sci. Rev. $128,599-627$.

Pätzold, M. et al., 2011. Asteroid 21 Lutetia: Low mass, high density. Science 334, 491-492. 
Preusker, F. et al., 2012. The Northern hemisphere of Asteroid 21 Lutetia Topography and orthoimages from Rosetta OSIRIS NAC image data. Planet Space Sci. 66, 54-63. http://dx.doi.org/10.1016/j.pss.2012.01.008.

Prockter, L., Thomas, P., Robinson, M., Joseph, J., Milne, A., Bussey, B., Veverka, J. Cheng, A., 2002. Surface expressions of structural features on Eros. Icarus 155 $75-93$.

Schulz, R., Sierks, H., Küppers, M., Accomazzo, A., 2012. Rosetta fly-by at Asteroid (21) Lutetia: An overview. Planet. Space Sci. 66, 2-8.

Sullivan, R., Greeley, R., Pappalardo, R., Asphaug, E., Moore, J.M., Morrison, D., Belton, M.J.S., Carr, M., Chapman, C.R., Geissler, P., Greenberg, R., Granahan, J., Head III, J.W., Kirk, R., McEwen, A., Lee, P., Thomas, P.C., Veverka, J., 1996. Geology of 243 Ida. Icarus 120, 119-139.

Thomas, P., Veverka, J., 1979. Grooves on asteroids: A prediction. Icarus 40, $394-$ 405.

Thomas, P., Veverka, J., Bloom, A., Duxbury, T., 1979. Grooves on Phobos - Their distribution, morphology and possible origin. J. Geophys. Res. 84, 8457-8477.

Thomas, P., Veverka, J., Bell, J., Lunine, J., Cruikshank, D., 1992. Satellites of Mars: Geologic history. In: Kieffer, H.H., Jakosky, B.M., Snyder, C.W., Matthews, M.S (Eds.), Mars. University of Arizona Press, Tucson.
Thomas, N., Stelter, R., Ivanov, A., Bridges, N.T., Herkenhoff, K.E., McEwen, A.S., 2010 Spectral heterogeneity on Phobos and Deimos: HiRISE observations and comparisons to Mars Pathfinder results. Planet. Space Sci. 59, 1281-1292. http://dx.doi.org/10.1016/j.pss.2010.04.018.

Thomas, N. et al., 2012. The geomorphology of (21) Lutetia: Results from the OSIRIS imaging system onboard ESA's Rosetta spacecraft. Planet. Space Sci. 66, 96-124. http://dx.doi.org/10.1016/j.pss.2011.10.003.

Tosi, F. et al., 2012. The light curve of Asteroid 21 Lutetia measured by VIRTIS-M during the Rosetta fly-by. Planet. Space Sci. 66, 9-22.

Veverka, J., Thomas, P., Simonelli, D., Belton, M.J.S., Carr, M., Chapman, C., Davies, M.E., Greeley, R., Greenberg, R., Head, J., 1994. Discovery of grooves on Gaspra. Icarus 107, 72-83.

Veverka, J. et al., 1997. NEAR's flyby of 253 Mathilde: Images of a C asteroid. Science 278, 2109-2112. http://dx.doi.org/10.1126/science.278.5346.2109.

Vincent, J.-B., Besse, S., Marchi, S., Sierks, H., the OSIRIS team, 2012. Physical properties of craters on Asteroid (21) Lutetia. Planet. Space Sci. 66, 79-86.

Weiss, B.P. et al., 2012. Possible evidence for partial differentiation of asteroid Lutetia from Rosetta. Planet. Space Sci. 66, 137-146. 